\title{
Acute Demyelinating Encephalomyelitis After Anti-venom Therapy in Russell's Viper Bite
}

\author{
S. Tripathy $\cdot$ P. K. Routray $\cdot$ A. K. Mohapatra • \\ M. Mohapatra $\cdot$ S C. Dash
}

Published online: 17 March 2010

(C) American College of Medical Toxicology 2010

\begin{abstract}
Introduction Russell's viper is a commonly encountered venomous snake in India. Morbidity and mortality following envenomation and the treatment thereof are frequent. We report a rarely seen complication after a treated Russell's viper bite.

Case Report A 36-year-old male farmer received 30 vials polyvalent anti-snake venom after a viper bite to his right leg. Improvement in initial hematemesis and circulatory shock was followed by acute renal failure managed with
\end{abstract}

Sources of Funding: None

S. Tripathy $(\bowtie)$

Department of Anesthesia and Intensive Care,

Kalinga Institute of Medical Sciences,

Patia,

Bhubaneswar, India

e-mail: swagi7@rediffmail.com

P. K. Routray

Department of Internal Medicine,

Kalinga Institute of Medical Sciences,

Patia,

Bhubaneswar, India

\section{A. K. Mohapatra}

Department of Neurology, Kalinga Hospital,

Bhubaneswar, India

M. Mohapatra

Kalinga Hospital,

Bhubaneswar, India

S. C. Dash

Department of Nephrology,

Kalinga Institute of Medical Sciences,

Bhubaneswar, India regular hemodialysis. He displayed no abnormalities on neurological examination at admission. Fourth day onwards his neurologic status started deteriorating with development of behavioral abnormalities, hemi-spatial neglect of left upper limb, paralysis of left facial nerve, left upper limb, and right lower limb. Acute disseminated encephalomyelitis was confirmed on magnetic resonance imaging (MRI) of brain with typical spectroscopic characteristics. High dose methyl prednisolone was administered and a rapid recovery followed.

Conclusion Russels viper bite followed by treatment with antivenom may be complicated by the development of immune complex mediated demyelination and development of acute disseminated encephalomyelitis. MRI spectroscopy helps in early identification of demyelination and in a definite diagnosis. Treatment with corticosteroids was associated with resolution of symptoms in this case.

Keywords Snake bite $\cdot$ Acute disseminated encephalomyelitis $\cdot$ Antivenom

\section{Introduction}

Acute disseminated encephalomyelitis (ADEM) is an autoimmune demyelinatimg disease of the nervous system which has only rarely been reported in literature to follow animal or insect bites, with a single reported case following a bite of an unknown snake [1,2]. We present a case of ADEM following a viper bite, in which recovery occurred after pulse methylprednisolone therapy. The role of early magnetic resonance imaging of brain and steroid therapy in the treatment of this unusual complication is discussed. 


\section{Case Report}

A 36-year-old previously healthy man, farmer by occupation, was bitten by a snake on the right calf. There was no history of any febrile illness, diarrhea, dysentery, malaria, or vaccinations in the recent past. There was no family history suggestive of psychiatric illness or multiple sclerosis. Patient was not addicted to tobacco or alcohol. The killed snake was identified as Russell's viper (Daboia russeli) in the nearest government hospital where he presented with hypotension and tachycardia (exact values at this time are not available to us). He received 30 vials of polyvalent Haffkine ${ }^{\circledR}$ anti-snake venom (manufactured by Haffkine Bio-pharmaceuticals Company, India) over a period of $12 \mathrm{~h}$ in the usual protocol of up to 30 vials in a $12 \mathrm{~h}$ period (ten vials at time (T) 0 , ten vials at $\mathrm{T} 0+6 \mathrm{~h}$, and ten vials at $\mathrm{T} 0+12 \mathrm{~h}$ ).

No neurological manifestations like ptosis, dyspnea, weakness, drowsiness, or disorientation were observed at presentation. Oozing from site of bite, bleeding from gums and hematemesis occurred. Coagulopathy was indicated by an abnormal $20 \mathrm{~min}$ whole bloodclotting time (20WBCT). 20WBCT is a simple bedside test in which low plasma fibrinogen concentration is indicated if $5 \mathrm{ml}$ fresh blood put in a clean dry glass test tube runs out when the tube is tipped once at $20 \mathrm{~min}$. This is repeated every $6 \mathrm{~h}$ until the blood clots, when the 20WBCT is said to be normal.

The patient remained hypotensive $(88 / 60 \mathrm{mmHg})$ and oliguric even after antivenom administration. Coagulopathy improved and 20WBCT normalized by $18 \mathrm{~h}$ of the bite. Deranged renal parameters and anticipated need for hemodialysis necessitated transfer to our center. This was approximately $24 \mathrm{~h}$ after the snake bite.

On examination at presentation to our hospital $24 \mathrm{~h}$ after the bite, patient was conscious and oriented. Pulse rate108 beats/min; bloodpressure $-90 / 60 \mathrm{mmHg}$, and respiration rate-26/min. Subconjunctival hemorrhage in the right eye and ecchymosis on the chest wall were present. Local examination at the site of viper bite showed hyperemia, tenderness, and tissue edema. Further administration of anti-snake venom (ASV) was withheld as the bleeding and clotting time was normal. Neurologic examination at this time was normal.

Investigations revealed hemoglobin $10.2 \mathrm{~g} / \mathrm{dl}$ (normal range $12-16 \mathrm{~g} / \mathrm{dl})$, platelets $1.5 \times 10^{5} / \mu \mathrm{l}\left(1.5-4 \times 10^{5} / \mu \mathrm{l}\right)$, total leucocyte count $9.2 \times 103 / \mu 1\left(4-11 \times 10^{3} / \mu 1\right)$, normocytic normochromic peripheral smear and normal coagulation profile (prothrombin time $15 \mathrm{~s}(13-15 \mathrm{~s})$, prothrombin time index $87 \%$, activated partial thromboplastin time $39 \mathrm{~s}(35-$ $40 \mathrm{~s})$ ). Serum electrolytes and liver function tests were normal. Renal parameters were serum urea $32 \mathrm{~g} / \mathrm{l}(20-30 \mathrm{~g} / \mathrm{l})$, serum creatinine $5.6 \mathrm{mg} / 1$ (0.8-1.2 mg/l). Renal parameters deteriorated over the next day and alternate day hemodialysis was started.
On day 4, patient suddenly developed motor weakness in the right lower limb and left upper limb. This progressed to paralysis by day 6 . Left seventh cranial nerve palsy (central type) and higher cortical functional abnormalities including hemispatial neglect, dysgraphia, acalculia, and anosognosia were seen.

Hemispatial neglect presented as denial of ownership of his left upper limb.

Intermittent behavior abnormalities such as incoherent speech and violence were observed over the following days. The delirium was associated with waxing and waning levels of consciousness. Patient had hallucinations of being attacked - sometimes by a snake. Pupils were equal and reacting to light. Ocular fundoscopy was unremarkable. Deep tendon reflexes were absent in the affected limbs.

Blood glucose, liver function tests, electrolytes, hematocrit, erythrocyte sedimentation rate, and urinalysis had been normal throughout the course of hospital stay. By the tenth day, renal functions normalized. The cerebrospinal fluid (CSF) obtained on day 6 of hospitalization was clear, with normal pressure, 15 lymphocytes $/ \mathrm{mm}^{3}, 70 \mathrm{mg} / \mathrm{dl}$ glucose, and $58 \mathrm{mg} / \mathrm{dl}$ protein. CSF oligoclonal bands were absent. Polymerase chain reaction analysis of CSF for herpes simplex virus (HSV) 1 and 2, and mycobacterium tuberculosis were negative. Venereal Disease Research Laboratories tests of CSF and blood were negative. Serologic studies for HIV, HSV, hepatitis viruses, and leptospira were negative. Blood and CSF cultures were negative for bacteria and fungi. Electroencephalography disclosed mild, generalized background slowing. A computed tomography of brain obtained on day 4 when the patient had developed motor weakness in right lower limb and left upper limb showed non-specific edematous changes in the left temporal lobe (Fig. 1).

MRI of brain (FLAIR and T2 weighted) was done on day 8 by which time motor weakness of affected limbs had progressed to paralysis and behavioral abnormalities had manifested. It showed multiple hyper intense lesions in sub cortical and deep white matter of lateral cerebral hemispheres (right more than left; Fig. 2).

Spectroscopy (Fig. 3) was characteristic of demyelination. MRI of cervical spine and nerve conduction tests done on the same day failed to reveal peripheral demyelination.

Based on the history, neurologic signs, MRI, and spectroscopy findings, a final diagnosis of ADEM was made. High dose methyl prednisolone $(1,000 \mathrm{mg} /$ day $)$ was administered intravenously in two divided doses per day for 5 days. This was followed by a tapering schedule of oral prednisone, tapered over the next 7 days. There was rapid improvement in sensorium with patient becoming calm and cooperative within 2 days. The motor power improved from grade 0 to 4 in left upper and right lower limbs over the next week. Ambulation with support was documented at discharge on day 20. 
Fig. 1 CT scan brain on day4. Edematous changes in right temporal lobe

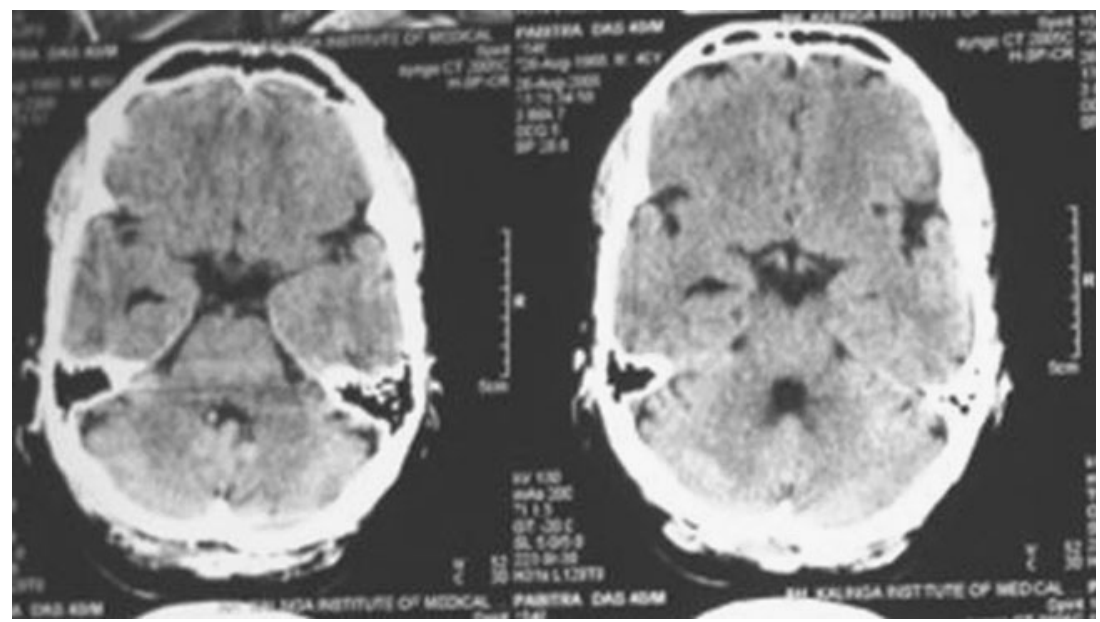

On follow-up at 3, 8, and 12 months, patient was doing well except for a tendency to occasionally neglect the left side of body like forgetting to don the left shoe. Patient was unwilling for a repeat MRI due to economic concerns.

\section{Discussion}

ADEM is a monophasic, demyelinating disease of the central nervous system of autoimmune origin characterized by an abrupt onset of neurologic symptoms preceded by an infection or recent immunization. It has been associated with infections such as measles, rubella, mumps, influenza, and herpes simplex. It may follow vaccination for rabies,

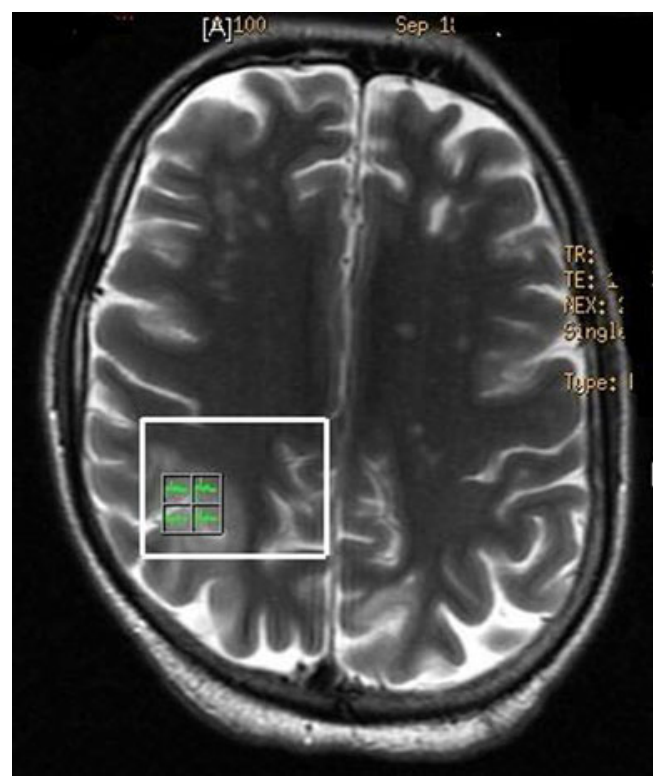

Fig. 2 MRI of brain (T2 weighted) showing multiple hyperintense lesions in subcortical and deep white matter of bilateral cerebral hemispheres. The area enclosed in the square demarcates the hyperintense region which has been spectroscopically analyzed tetanus, Japanese encephalitis, hepatitis B, and typhus [3]. It has been known to follow bee stings [1].

D. russelli is listed by World Health Organization as a snake of class 1 (commonly cause death or serious disability) among Indian snakes of medical importance [4]. Acute renal failure is a well-known entity in Russell's viper envenomation, the exact pathogenesis of which is not well established. However, a number of factors may contribute viz. bleeding, hypotension, circulatory collapse, intravascular hemolysis, disseminated intravascular coagulation, microangiopathic hemolytic anemia, and also direct nephrotoxicity of the venom [5]. The time course of events

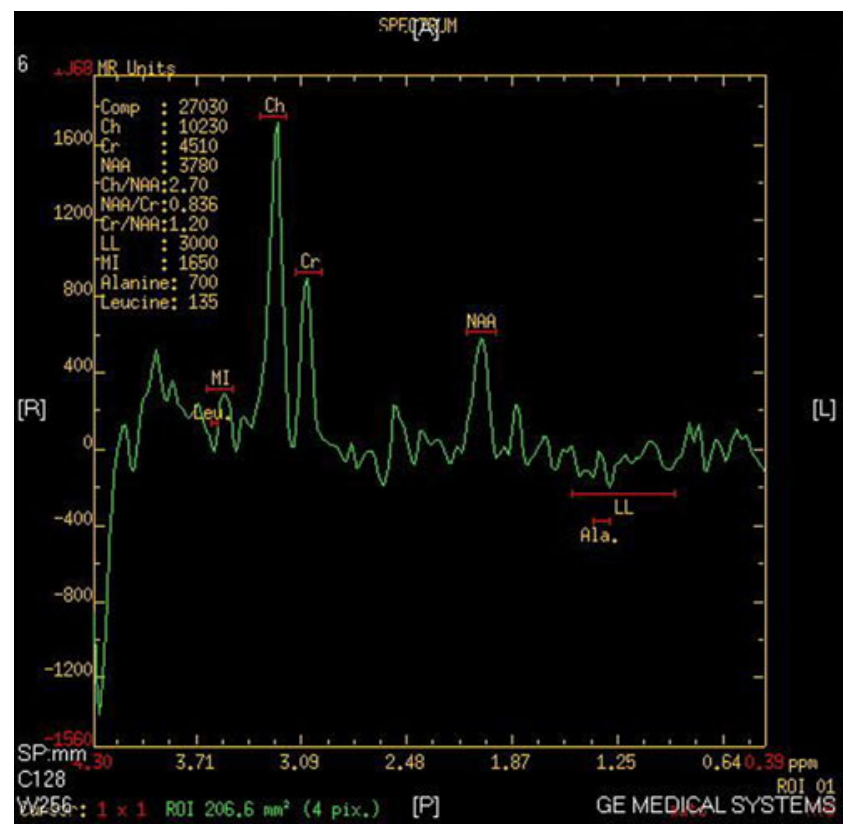

Fig. 3 Spectroscopy of affected area revealing slightly reduced Nacetyl aspartate, normal creatinine, and high choline peaks. NAA Nacetyl aspartate, $C h$ choline, $C r$ creatine, $M I$ myoinisitol, $L L$ lactate/ lipids, Leu leucine, Ala alanine 
and eventual complete recovery would suggest acute tubular necrosis as the possible pathology in our case.

Neurological symptoms after Russell's viper envenomation like ptosis, respiratory distress, and intracranial hemorrhage are experienced usually within $6 \mathrm{~h}$ after the bite as per our experience and as published elsewhere [6].

Indian polyvalent ASV is an equine product made with Naja naja, Bungarus caeruleus, D. russelii, and Echis carinatus venoms. Adverse reactions to antivenom appear in two forms; early and late. Early reactions like anaphylaxis occur in less than $10 \mathrm{~min}$ to $180 \mathrm{~min}$ after treatment. Late reactions are immune complex diseases and present in the form of serum sickness syndrome usually 5-24 days after anti-venom administration [7]. In view of delayed onset, we postulate some form of immune mediated neuropathy due to the ASV as a more likely cause of ADEM than direct neurotoxicity of snake venom in our case.

The differential diagnosis of ADEM is extensive. It is difficult to differentiate ADEM from an initial presentation of multiple sclerosis - the second attack in those who progress to MS usually occurs within 1 year. Many consider the two to be a part of a spectrum of inflammatory demyelinating conditions [3]. Absence of electrolyte disturbances during the entire course of stay would rule out reversible osmotic demyelination syndrome associated with hemodialysis. Other multifocal disease processes that give rise to hyperintense T2-weighted lesions on MRI have appearances, locations, and spectroscopy findings that are not typical of ADEM.

In the only other such case described by Malhotra et al [2], the snake had not been identified (snakes with predominantly neurotoxic venoms of the Elapidae family are more common than vipers in the northern parts of India from where this report comes). There is no mention of bleeding or coagulopathy having developed. Motor power improved with ASV (100 $\mathrm{ml} 8$ hourly) initially and deteriorated again after a week. The patient presented to them after 10 days of bite with advanced renal failure and severe motor weakness. ASV treatment was continued. The computed tomography (CT) scan demonstrated diffuse white matter hypointensity and patient showed no improvement with high dose corticosteroids or plasmapheresis. Diagnosis was confirmed with post mortem biopsy.

Magnetic resonance spectroscopy (MRS) complements MRI as a non-invasive means for the characterization of tissue. While MRI uses the signal from hydrogen atoms to form anatomic images, proton MRS uses this information to determine the concentration of brain metabolites such as $\mathrm{N}$-acetyl aspartate, choline, creatine, and lactate in the tissue examined. The most widely used clinical application of MRS has been in the evaluation of central nervous system disorders. A specific pattern of metabolites can be seen in different pathologic states, like untreated bacterial brain abscess, brain tumors, or areas of demyelination [8]. Recent imaging studies with enhanced-magnetic resonance imagers and spectroscopy have defined a characteristic pattern in demyelination with slightly reduced $\mathrm{N}$-acetyl aspartate, normal creatine peaks, and high choline peak as documented in our case (Fig. 3). In the future, magnetic resonance imaging findings may give better data on nonfatal, nonbiopsied cases of suspected post-snake bite or ASV-related ADEM.

High-dose corticosteroids are the treatment of choice in inflammatory, demyelinating CNS diseases. Methylprednisolone $10-30 \mathrm{mg} / \mathrm{kg} / \mathrm{d}$ (maximum $1 \mathrm{~g} /$ day) over 3-5 days followed by a tapered oral prednisolone over 2-6 weeks, is recommended as a standard medical treatment [9]. Other options include the use of anti-inflammatory and immunosuppressive treatments such as intravenous immunoglobulin and plasmapheresis specially when no improvement is seen or the patient continues to deteriorate [10].

Delayed neurologic manifestations in a case of treated Russell's viper envenomation should be investigated with MRI and spectroscopy for ADEM. High-dose methylprednisolone therapy appeared to be effective in our case and should be considered in proven cases of ADEM following envenomation and treatment of Russell's viper bite.

\section{References}

1. Boz C, Velioglu S, Ozmenoglu M (2003) Acute disseminated encephalomyelitis after bee sting. Neurol Sci 23:313-315

2. Malhotra P, Sharma N, Awasthi A, Vasishta RK (2005) Fatal acute disseminated encephalomyelitis following treated snake bite in India. Emerg Med J 22:308-309

3. Garg RK (2003) Acute disseminated encephalomyelitis. Postgrad Med J 79:11-17

4. Simpson ID, Robert NL (2006) Snakes of medical importance in India: is the concept of the "Big 4" still relevant and useful? Wilderness Environ Med 18:2-9

5. Kohli HS, Sakhuja V (2003) Snake bites and acute renal failure. Saudi J Kidney Dis Transpl 14:165-76

6. Seneviratne U, Dissanayake S (2002) Neurological manifestations of snake bite in Sri Lanka. J Postgrad Med 4:275-279

7. Warrell D (1996) Injuries, envenoming, poisoning, and allergic reactions caused by animals. In: Weatherall DJ, Ledingham JGG, Warrell DA (eds) Oxford textbook of medicine, 3rd edn. Oxford University Press, Oxford, pp 1124-51

8. Gujar SK, Maheshwari S, Björkman-Burtscher I, Sundgren PC (2005) Magnetic resonance spectroscopy. J Neuroophthalmol 25 (3):217-26

9. Dale RC, Branson JA (2005) Acute disseminated encephalomyelitis or multiple sclerosis: can the initial presentation help in establishing a correct diagnosis? Arch Dis Child 90:636-639

10. Mihai C, Jubelt B (2005) Post-infectious encephalomyelitis. Curr Neurol Neurosci Rep 5:440-445 